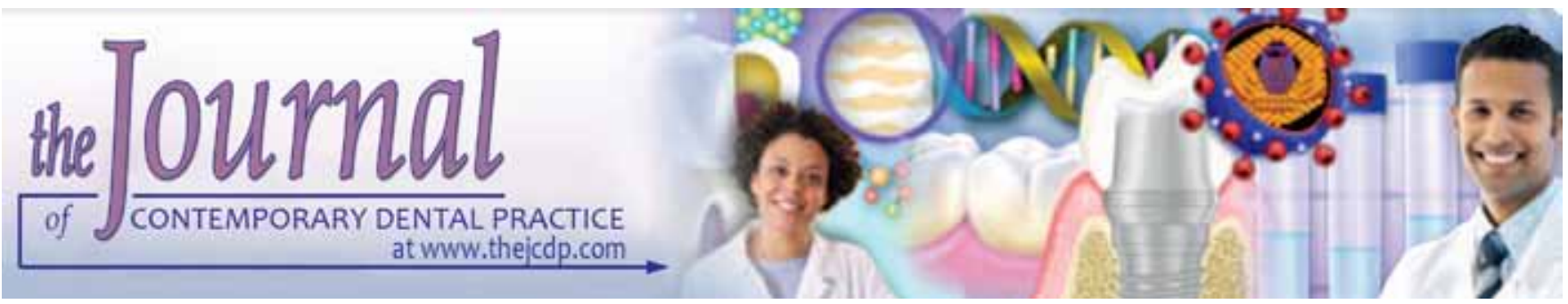

\title{
Management of Maxillary Deformity with Segmental Osteotomy followed by Implant Insertion in $\beta$-Thalassemia Major Patient
}

'Zafer Ozgur Pektas, ${ }^{2}$ Secil Cubuk, ${ }^{3}$ Beyza Kircelli, ${ }^{4}$ Sina Uckan

\begin{abstract}
Aim: The aim of this report is to present the management of the maxillary deformity and subsequent implant therapy of a case with $\beta$-thalassemia major.
\end{abstract}

Background: $\beta$-thalassemia is a hematologic disorder that results from the abnormality of the $\beta$-globulin chain synthesis. The best known thalassemia-induced dentofacial problem is the maxillary enlargement, and this undesirable growth of maxilla affects not only the facial esthetics but also dental occlusion, and leads to functional deficiency.

Case description: A 16-year-old female patient with $\beta$-thalassemia major was referred with the complaints of severe facial deformity and malocclusion, resulting in psychosocial and functional problems for her. The dentofacial deformity was characterized by an excessive premaxillary growth both in sagittal and vertical planes. Anterior maxillary osteotomy was performed with bilateral canines extraction, and dental implants were inserted to the canine regions, following bone healing. Postoperative course was free of problems with the crown restorations in function. Recurrence has not been occurred at 6 years follow-up.

Conclusion: With maintaining hemoglobin level over $10 \mathrm{gm} / \mathrm{dl}$, correction of maxillary defects is stable for long-term in $\beta$-thalassemia major patient. Implant application to these patients may lead to unforeseeable results.

${ }^{1}$ Department of Oral and Maxillofacial Surgery, Baskent University, Adana, Turkey

${ }^{2}$ Department of Oral and Maxillofacial Surgery, Faculty of Dentistry, Medipol University, Istanbul, Turkey

${ }^{3}$ Department of Orthodontics, Private Clinic, Adana, Turkey

${ }^{4}$ Department of Oral and Maxillofacial Surgery, Medipol University, Istanbul

Corresponding Author: Secil Cubuk, PhD, Department of Oral and Maxillofacial Surgery, Faculty of Dentistry Medipol University, Istanbul,Turkey, Phone: 902124401010 e-mail:secilcubuk@hotmail.com
Clinical significance: Although having some difficulties, such as overbleeding and stability problems, maxillary enlargement can be treated by segmental osteotomies successfully in $\beta$-thalassemia major patient. Implant failure frequency may be higher, but many other studies are needed for determining implant survival rate in $\beta$-thalassemia major patients.

Keywords: Correction of malocclusion, Hematologic disorder, Implants, Orthognathic surgery, $\beta$-thalassemia.

How to cite this article: Pektas ZO, Cubuk S, Kircelli B, Uckan S. Management of Maxillary Deformity with Segmental Osteotomy followed by Implant Insertion in $\beta$-Thalassemia Major Patient. J Contemp Dent Pract 2015;16(8):704-707.

Source of support: Nil

Conflict of interest: None

\section{INTRODUCTION}

$\beta$-thalassemia is a hematologic disorder that results from the abnormality of the $\beta$-globulin chain synthesis. Although thalassemia is the most frequent monogenic diseases worldwide, the highest prevalence is in the Mediterranean region, particularly in Greece, Turkey, and Cyprus, and in middle-east countries, such as Iran, Qatar and Jordan. ${ }^{1}$

Individuals with $\beta$-thalassemia are transfusiondependent, and with early diagnoses and treatment inception they can survive for many years. However, chronic anemia induces the absorption of iron from gastrointestinal system and multiorgan systems can be impaired due to the iron deposition. Eventually, cardiac failure and endocrine abnormalities can develop because of iron overloading at such organs. ${ }^{2}$ Another systemic complication of the disease is hypercoagulability with strong evidence about coagulation system activation in $\beta$-thalassemia. ${ }^{3}$ 
The best known thalassemia-induced dentofacial problem is the maxillary enlargement. ${ }^{4}$ Underproduction of $\beta$-globulin chains lead to increase of free e $\alpha$-globulin chains, that are normally paired with $\beta$-globulin chains and compose the hemoglobin structure. Unstable free $\alpha$-globulin chains form precipitates within the red cells and destroy the bone marrow site. As a consequence, compensatory extramedullary erythropoiesis takes place, and maxillary and the other skull and facial bone deformities may appear. ${ }^{1,2}$

Enlargement of the maxillary bone affects not only the facial esthetics, but also dental occlusion and leads to functional deficiency. However, there are a few reports about maxillary correction surgery in the literature and there is only one case report of implant application to the thalassemia minor patient. ${ }^{2,5-9}$

This report presents the management of the maxillary deformity and subsequent implant therapy of a case with $\beta$-thalassemia major.

\section{CASE REPORT}

A 16-year-old female patient with $\beta$-thalassemia major was referred with the complaints of severe facial deformity and malocclusion, resulting in psychosocial and functional problems for her. The dentofacial deformity was characterized by an excessive premaxillary growth both in sagittal and vertical planes (Figs 1A and B). Maxillary incisors were protruded with an extreme overjet and overbite, causing an apparent gummy smile and lip incompetence. Her medical history revealed multiple transfusions from the age of 1.5 to 9 , when she had underwent a prophylactic splenectomy. The dental treatment was commenced with orthodontic alignment. The treatment plan comprised of bilateral maxillary first bicuspid extractions for the retraction of the maxillary incisors on the basis of maximum anchorage. The bilateral maxillary canines were retracted; however, the uprighting of these teeth could not be accomplished despite all the attempts throughout a year. Finally, it was decided to extract the bilateral canines intraoperatively to maintain an adequate space for the maxillary segmental osteotomy. The treatment course proceeded with the surgery and she was prepared for the operation. The preoperative hemoglobin level was detected as $12.40 \mathrm{gm} / \mathrm{dl}$ and there was no requirement of preoperative transfusion. Cardiological and hematological consultations revealed no disability for surgery and general anesthesia, such as cardiac dysfunction or hemorrhage diathesis.

\section{Surgical Protocol}

The general anesthesia was administered via the nasotracheal intubation. After infiltrating the facial mucosa with the local anesthesia (2\% lidocaine with $1 / 100,000$ epinephrine), the horizontal mucosal incision was performed between the bilateral maxillary first bicuspids, superior to the mucogingival junction. Subsequent to the extraction of bilateral maxillary canines due to the aforementioned indications, the anterior maxillary osteotomy line was exposed by mucoperiosteal dissection, and the bilateral vertical osteotomy lines were exposed by coronal tunneling up to the crests of the extraction sites. The horizontal osteotomy was carried out staying about $4 \mathrm{~mm}$ above root apices and vertical osteotomies were performed at the level of canine teeth with saw. After completing the palatal osteotomy, the anterior maxillary segment was mobilized and set back about $10 \mathrm{~mm}$. The anterior maxillary segment was fixed with two L plates and miniscrews (Synthes, J and J Company). Finally, the bone removed during the maxillary ostectomy was used as autogenous graft in the superior osteotomy gap to facilitate the osseous healing. The incision line was sutured with 3/0 vicryl. Antibiotic, anti-inflammatory and antibacterial mouthwashes were prescribed to the patient. The healing was uneventful and postsurgical

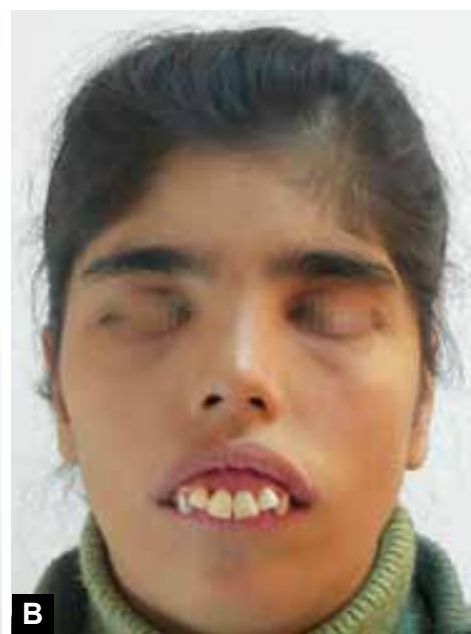

Figs 1A and B: (A) Preoperative sagittal view; excessive premaxillary growth and lip incompetence was detected and (B) preoperative frontal view 

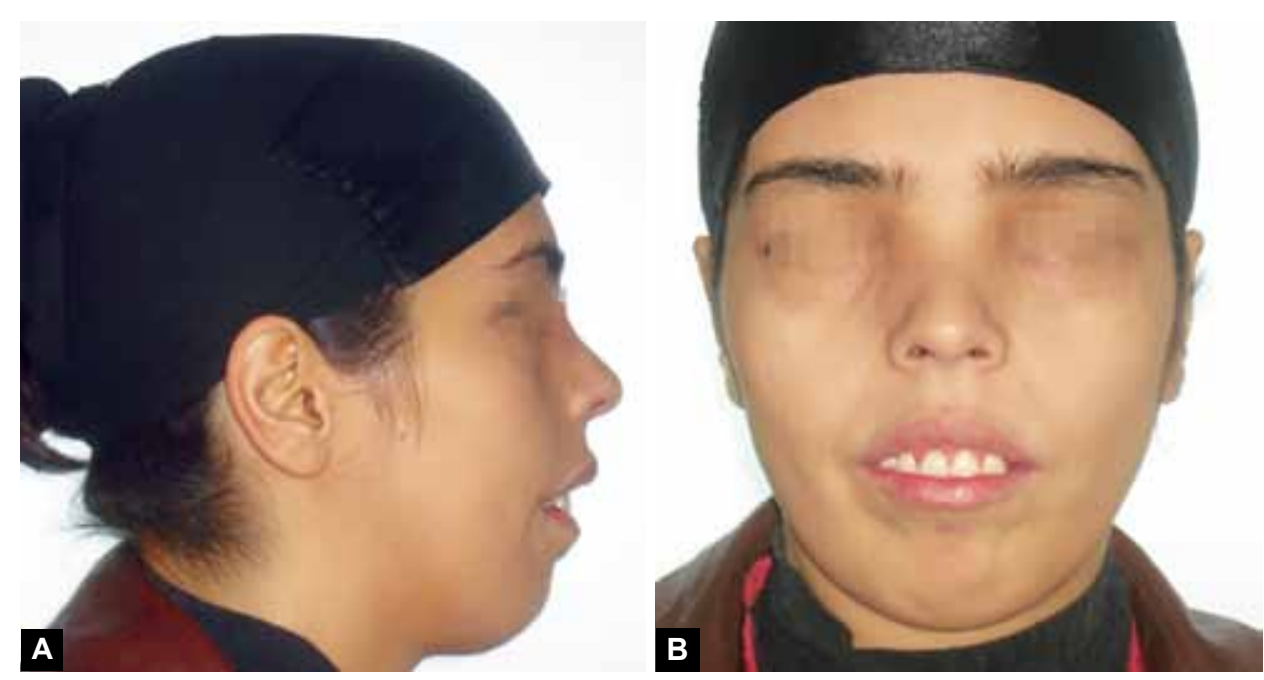

Figs 2A and B: (A) Postoperative sagittal view and (B) postoperative frontal view

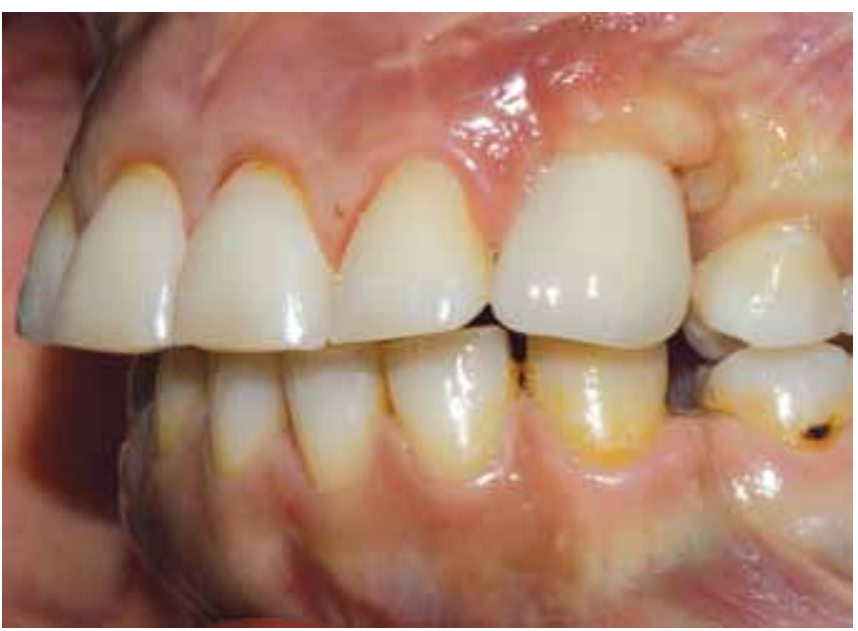

Fig. 3: Intraoral viewing of the left canine crown restoration and its occlusal statement

orthodontic treatment was carried out for postoperative 3 years (Figs $2 \mathrm{~A}$ and $\mathrm{B}$ ). The clinical results of the orthodontic treatment enabled an adequate space for the placement of two dental implants (Zimmer Dental, US, $3.7 \mathrm{~mm}$ width and $13 \mathrm{~mm}$ length) to the bilateral maxillary canine regions. The implants were loaded after 8 months and restored with two individual crowns. Unfortunately, the left implant was lost at the 5th month of loading. After allowing for an adequate bone healing period of 4 months, another implant was placed into this region. The time elapse between the insertion and loading of the implant was 6 months. The relatively long periods for the implants to be osseointegrated were determined regarding the impaired osseous healing. Postoperative course was free of problems with the crown restorations in function (Fig. 3).

\section{DISCUSSION}

$\beta$-thalassemia major is a hemoglobinopathy that is seen with skeletal and facial bone abnormalities. Skeletal deformities, such as growth retardation with earlier fusion of long bones' epiphysis, vertebrae, pelvis and chest deformities have been described in $\beta$-thalasemia major. ${ }^{10}$ Most of the thalassemic patients have class II malocclusion with protrusive premaxilla, prominent cheek bones and frontal bones and depressed nose. ${ }^{4}$

Earlier transfusion treatment and maintenance of hemoglobin greater than $10 \mathrm{gm} / \mathrm{dl}$ is important for preventing the skeletal deformities like maxillary enlargement. ${ }^{8}$ In this case, despite transfusion therapy hemoglobin level had not always maintained over $10 \mathrm{gm} / \mathrm{dl}$ at preoperative term. Following the operation, the hemoglobin level has mostly been over $10 \mathrm{gm} / \mathrm{dl}$; therefore, maxillary growth and recurrence has not been occurred at 6 years follow-up.

Surgical management of dentofacial deformities in $\beta$-thalassemia major patients has many challenges in preoperative, intraoperative and postoperative term. There are few studies in correction of maxillary deformities with surgical resection, contouring and Le Fort I osteotomy in thalassemic patients. ${ }^{2,5-9}$ To the best of our knowledge, there is only one study about reconstruction of the maxilla with anterior segmental osteotomy in thalassemia major patient. ${ }^{5}$ In this report, anterior maxilla reposition was performed both vertically and sagittally.

Because of iron overloading, multisystem organ failure, such as cardiovascular and endocrine system disease can be seen in these patients. This presented case did not have any cardiac or other system disease; just underwent splenectomy for a symptomatic enlarged spleen.

Massive bleeding can make surgery difficult and can be a hurdle in performing maxillary and mandibular osteotomies. It was reported that 11.8 liters blood loss had occurred because of overgrown bone marrow while performing maxillary osteotomies in a $\beta$-thalassamia patient. ${ }^{9}$ It is important to get prepared for severe bleeding and use of intraoperative blood loss salvage, such as hypotensive anesthesia and blood transfusion protocol. 
However, in the presented case minor blood loss occurred and there was no need of blood transfusion.

Although fixation problems may be encountered in patients with $\beta$-thalassemia major due to the thin cortical plates and jaws that are rich of bone marrow, we did not face any difficulties in fixation. In this case, adequate fixation was maintained with two L-shaped mini plates and eight miniscrews.

An adequate primary stability of two implants was achieved; and therefore, they were loaded following 8 months of their placement. One implant failed and was removed at 5 th month following its loading. After 4 months, another implant was inserted to the same socket and it survived at the 6 months control appointment.

There was only one report about implant surgery in $\beta$-thalassemia case in literature; however, that was the minor type. ${ }^{10}$ In that report, eight implants had been inserted to maxilla using tapered osteotomes for minimum bone removal. Within more than 1-year-follow-up, no implants had been lost. This is the first report about implant application in $\beta$-thalasemia major. One implant was extracted in this case, although there was not any marginal bone loss or signs of infection. It must always be taken into consideration that maxilla has poorer quality bone and thinner cortical plate in $\beta$-thalassemia major than minor type, so implant success and survival can be lower. Besides this, there is a requirement of more studies that have more cases and long-term clinical follow-up.

\section{CONCLUSION}

We have concluded that $\beta$-thalassemia major is a hemoglobinopathy that can lead to deformities at facial bones, particularly at maxilla. Although surgery in these patients has many difficulties, with hemoglobin level over $10 \mathrm{gm} / \mathrm{dl}$, correction of maxillary defects is a stable for long-term. Implant application to these patients may lead to unforeseeable results.

\section{CLINICAL SIGNIFICANCE}

There are some reports regarding orthognathic surgery in $\beta$-thalassemia major patients, but this is the first case report about implant application and maxillary segmental osteotomy in one such patient. Although having some difficulties, such as overbleeding and stability problems, maxillary enlargement can be treated by segmental osteotomies successfully. Implant failure frequency may be higher but many other studies are needed for determining implant survival rate in $\beta$-thalassemia major patients.

\section{REFERENCES}

1. Bassimitci S, Yücel-Eroğlu E, Akalar M. Effects of thalasaemia major on components of the craniofacial complex. Brit J Orthod 1996;23(2):157-162.

2. Park N, Lazow S, Berger J. $\beta$-thalassemia: medical and surgical consideration in managing facial deformities: case report and review of the literature.J Oral MaxillofacSurg 2012;70(4):284-289.

3. Cappellini DM, Motta I, Musallam KM, Taher AT. Redefining thalassemia as a hypercoagulable state. Ann NY Acad Sci 2010:1202;231-236.

4. Sackey K. Hemolytic anemia: part 2. Ped Rev/Am Acad Pediatr 1999;20(6):204-208.

5. Jurkiewics MJ, Pearson HA, Furlow LT. Reconstruction of the maxilla in thalassemia. Ann NY Acad Sci 1969;165(1):437-442.

6. Weel F, Jackson IT, Crookendale WA, McMichan J. A case of thalassemia major with gross dental and jaw deformities. Br J Oral Maxillofac Surg 1987;25(4):348-352.

7. Drew SJ, Sachs SA. Management of the thalassemia-induced skeletal deformity: case report and review of the literature. J Oral Maxillofac Surg 1997;55(11):1131-1139.

8. Mortazavi SH, Khojasteh A. Superior repositioning of the maxilla in thalassemia-induced facial deformity: report of 3 cases and a review of the literature. J Oral Maxillofac Surg 2007;65(5):1023-1031.

9. Mak PH, Ooi RG. Submental intubation in a patient with beta-thalassaemia major undergoing elective maxillary and mandibular osteotomies. Br J Anaesth 2002;88(2):288-291.

10. Misch CM,Jolly RL, Williams DR, Chorazy CJ.Maxillary implant surgery on a patient with thalassemia: a case report. Oral Surg Oral Med Oral Pathol Oral Radiol Endod 1998;86(4): 401-405. 